\title{
Modulation of proliferation, apoptosis and inflammation of Caco-2 epithelial cells and THP-1 macrophage-like monocytes in LPS stimulated co-culture model
}

\author{
Biriken $\mathrm{D}^{1}$, Yazihan $\mathrm{N}^{2,3}$
}

Ankara University, Faculty of Medicine, Department of Pathophysiology, Turkey.nyazihan@ankara.edu.tr

\begin{abstract}
BACKGROUND AND OBJECTIVES: Epithelial cells and macrophages play major roles in modulating the state of inlammatory response regulated by intracellular signaling pathways. The aim of this study was to characterize changes in cell proliferation, apoptosis and inflammation related intracellular signalling pathways MAPKs and NF-KB in Caco-2 epithelial cells and THP-1 macrophage-like monocytes contacted and filterseperated co-cultures in the presence of LPS stimulation.

METHODS: We assessed the apoptosis and inflammation by measuring caspase- 3 activity, TNF- $\alpha$ and IL-10 cytokines, total and phosphorylated forms of intracellular signalling pathway molecules p53, JNK, Jun, ERK, p38, NF-kB p65 and IkB.

RESULTS: The contacted co-culture of Caco-2 and THP-1 cells represented higher levels of JNK, jun and p38 MAPK pathway proteins associated with cell proliferation, whereas apoptosis related molecule caspase-3 and p53 increased in the filter-separated co-culture condition. Also, the contacted co-culture condition led to proinflammatory changes in NF-KB signalling pathways and cytokine responses with high phosphorylated NFKB p65 and TNF- $\alpha$, and low I-KB and IL-10 levels.

CONCLUSION: Epithelia - monocytes co-culture stimulated with LPS regulated cell proliferation and inflammation in a contact dependent manner, whereas apoptosis was associated with non-contacted cell culture condition. Thus, co-culture models are important models for explaining the immunopathologies in the mucosal areas (Fig. 5, Ref. 30). Text in PDF www.elis.sk

KEY WORDS: Caco-2 epithelial cells, THP-1 monocytes, co-culture, lipopolysaccharide (LPS), NF-kB, MAPK.
\end{abstract}

\section{Introduction}

Due to their strategical anatomical arrangement, intestinal epithelial cells are not just a physical barrier, they are also unique regulators in the maintenance of mucosal homeostasis through playing an active role in the interaction between bacteria and immune cells at the lamina propria. The interaction between epithelial and immune cells is complex and has not been well appreciated yet. The intestinal epithelial cells transform the signals from bacteria to immune cells of the lamina propria, thus maintain the coordinated immune response (1-3). Macrophages, considered the first cells to come in contact with the pathogens after passing the epithelial barrier, which allow the sustained innate immune and inflammatory response

${ }^{1}$ Ankara University, Faculty of Medicine, Department of Microbiology, Turkey, ${ }^{2}$ Ankara University, Institute of Health Sciences, Interdisciplinary Food, Metabolism and Clinical Nutrition Department, Turkey, and ${ }^{3}$ Ankara University, Faculty of Medicine, Department of Pathophysiology, Turkey

Address for correspondence: N. Yazihan, Ankara University, Faculty of Medicine, Internal Medicine, Pathophysiology Department, Morfoloji Building, Sihhiye, Ankara 06100,Turkey.

Phone: +90.312 .5958056$

Grant support: This research was supported by The Scientific and Technological Research Council of Turkey (TUBITAK). against pathogens at the site of infection $(4,5)$. The interaction between these two important cells of the innate immune response, epithelial cells and monocytes, occur in epithelium. Thus, the interaction of these two effector cells stimulates the intracellular signaling pathways responsible for triggering the immune response $(6,7)$.

Mitogen-activated protein kinase (MAPK) and nuclear factor$\kappa \mathrm{B}(\mathrm{NF}-\kappa \mathrm{B})$ signalling cascade proteins have been demonstrated to play a major role in immune response by mediating extracellular signals to cellular responses $(8,9)$. So far, three MAPK families have been identified in mammalian cells, the extracellular signalregulated kinase (ERK), Jun kinase (JNK) and p38 MAPK. A variety of stimuli can lead to the activation of these cascades that elicits a vital role in immune cell proliferation, differentiation, inflammatory responses and apoptosis $(8,10)$. NF- $\mathrm{KB}$ represents a family of inducible transcription factors, which are normally sequestered in the cytoplasm by a family of inhibitory proteins, including I $\mathrm{KB}$ family members. The transcription factor NF- $\kappa \mathrm{B}$ regulates the survival, activation and differentiation of immune cells, thus after activation with different stimuli they orchestrate the immune and inflammatory responses including cytokine responses (11). Signal transduction of MAPK and NF- $\kappa$ B results in activation via phosphorylation, while MAPK phosphorylation can be used as a measure of the pathway activity $(12,13)$. 
Cytokine network and intercellular contact are crucial in immune cell activation in the mucosa. The cytokines lead to complex cellular interactions and reciprocal messaging between the epithelia and macrophages. Pro-inflammatory cytokines like TNF- $\alpha$ are produced both from the epithelial cells and the macrophages. Whereas, IL-10 is released only from active macrophages, not only has a strong anti-inflammatory property (14) through the inhibition of inflammatory cytokines but also is known to protect the intestinal epithelial barrier function $(15,16)$.

The aim of this study was to characterize the changes in the protein expressions related to cell proliferation, apoptosis and inflammation with MAPK and NF- $\mathrm{kB}$ activation and signalling in Caco-2 epithelial cells - THP-1 monocyte-like cells co-culture model.

\section{Materials and methods}

\section{Cell cultures and co-culture model}

Caco-2 epithelial cells (colon epithelial adenocarcinoma cell) (DSMZ; ACC169, Braunschweig, Germany) and non-adherent THP-1 macrophage-like monocytes (human leukemia monocytic cell) (DSMZ; ACC-16, Braunschweig, Germany) were grown in RPMI 1640 medium (Sigma, USA), supplemented with 10 $\%$ FCS, $100 \mathrm{IU} / \mathrm{ml} \mathrm{L-glutamine} \mathrm{(Sigma,} \mathrm{USA),} \mathrm{and} 100 \mathrm{mg} / \mathrm{ml}$ penicillin-streptomycin (Sigma, USA) in tissue culture flasks. The experiments were done in a humidified atmosphere of $5 \%$ $\mathrm{CO}_{2}$ in air at $37{ }^{\circ} \mathrm{C}$. For use in the experiments, a suspension of $1 \times 10^{5}$ cells $/ \mathrm{ml}$ was prepared in culture medium and seeded in six well plates (Nunc, Denmark) as four different conditions such as; i. Co-culture of Caco-2 epithelial cells and THP-1 macrophagelike monocytes separated with a cell separator (Thincert W. Petmembrane, $0.4 \mu \mathrm{m}$; Greiner, Germany), ii. Contacted co-culture of Caco-2 and THP-1 cells, iii. single cell culture of Caco-2 cells and iv. single cell culture of THP-1 cells (17). After three hours of incubation for adherence, the cells were treated with $1 \mu \mathrm{g} / \mathrm{ml}$ of LPS ( $E$. coli O111) and incubated in a humidified atmosphere of $5 \% \mathrm{CO}_{2}$ at $37{ }^{\circ} \mathrm{C}$ for 24 hours. After incubation, cell lysates and supernatants were collected from the wells and they were kept at $-80{ }^{\circ} \mathrm{C}$ until further use.

\section{MTT assay}

The changes in cell proliferation of the living cells were measured through the absorbance of the 3-(4,5-dimethylthiazol2-yl)-2,5-diphenyltetrazolium bromide dye (MTT; Sigma, USA) which is based on the tetrazolium ring of the MTT dye being broken down by the mitochondria of the viable cells (18). The cells were dispended to 96 well plates and left for 24 hour incubation. Then LPS was added onto the wells and incubated for another 24-48 hours in $5 \% \mathrm{CO}_{2}$ atmosphere at $37^{\circ} \mathrm{C}$. After the incubation period, $100 \mu 1$ of $10 \%$ MTT dye $(5 \mathrm{mg} / \mathrm{ml}$ in PBS) was added to each well and the plates were incubated for more 4 hours. Then $100 \mu 1$ of $0.08 \mathrm{M} \mathrm{HCl}$ containing absolute isopropanol was added onto each well and dissolved formazan absorbance was measured in the spectrophotometer (Epoch Microplate Spectrophotometer, Biotek Company, USA) at $540 \mathrm{~nm}$.

\section{Determinations of apoptosis by caspase-3}

Caspase- 3 was measured to evaluate apoptosis. The cells were spread out onto 96 well plates and incubated for 24 hours. Then LPS was added onto the wells and incubated in $5 \% \mathrm{CO}_{2}$ atmosphere at $37{ }^{\circ} \mathrm{C}$ for $24-48$ hours. At the end of the experiment, the cells were disrupted with assay buffer (50 mM HEPES, $\mathrm{pH}$ 7.4, $100 \mathrm{mM} \mathrm{NaCl}, 0.1 \%$ CHAPS, 10 mM DTT, 2 mM EDTA, 2 mM EGTA, $0.1 \%$ Triton X-100). The excitation emission wave lengths of 7-amino-4-methylcoumarin (AMC) were $360 \mathrm{~nm}$ and $460 \mathrm{~nm}$.

Proteins of the intracellular signalling pathways and cytokine secretions

Nuclear and cytosolic fractions were prepared according to protocol of Bijur and Jope (19). Briefly, the cells were washed with phoshate buffered saline (PBS) and lysis buffer (CelLytic NuCLEAR Extraction Kit, Sigma), phosphatase inhibitors and protease inhibitors were added to cells. Nuclear fraction of cell lysates was obtained by centrifugation for $15 \mathrm{~min}$ at $4{ }^{\circ} \mathrm{C}$ according to kit procedure. Protein concentrations in the cytosolic and nuclear extracts were determined using the bicinchoninic acid method (Sigma, USA).

Cell culture supernatants were harvested in lysis buffer and analyzed for proinflammatory cytokine TNF- $\alpha$, anti-inflammatory cytokine IL-10 (E-BIOSCIENCE), total and phosphorylated forms of intracellular signalling pathway molecules; p38, JNK, ERK, Jun, IkB, p53 and NF- $\kappa$ B p65 (eBiosciences Platinium ELISA kits, Thermo Scientific). The cytokine levels were measured in triplicates using commercial ELISA kits.

\section{Statistical analysis}

Graphpad Prism 7.03 (Graphpad software, La Jolla, CA, USA) was used for all statistical analyses. For multiple group comparisons, Kruskal-Wallis non-parametric tests, one-way ANOVA with Dunn's test as post hoc were applied. Single comparisons of independent groups were compared using the non-paired MannWhitney $U$ test $p$ values $<0.05$ were regarded as significant, and the results of the statistical analysis are indicated in the Figures as $* \mathrm{p}<0.05$.

\section{Results}

\section{Cell proliferation of epithelial and monocyte cells in co-culture} model

In this study, the effect of LPS stimulation in cell proliferation of Caco-2 epithelia and THP-1 monocytes in a co-culture model were evaluated with MTT after 24-48 hours of treatment. No significant difference was seen in the co-culture model compared to the monolayer cell lines, neither at 24 hours nor at 48 hours (data not shown).

\section{Apoptosis of epithelial and monocyte cells in co-culture model}

The effect of LPS treatment on apoptosis of Caco-2 epithelia and THP-1 monocyte co-culture model was assessed by measuring the activities of p53 and caspase-3 (Figs 1 and 2). Both total and 



Fig. 1. The effect of LPS treatment on p53 in Caco-2 epithelia and THP-1 monocyte co-culture model. Activation of (A) total p53 and (B) phosphorylated p53 on different co-culture conditions after 24 hours of LPS stimulation. The horizontal bars indicate the medians of the results within each column. The results of the statistical analysis are indicated as $* \mathbf{p}<0.05$.

phosphorylated p53 showed slightly higher levels after 24 hours of LPS stimulation compared to non-stimulated culture conditions (data not shown). After LPS treatment, THP-1 monocyte cells represented higher levels of p53 in co-culture model without cell-cell contact, whereas Caco-2 epithelial cells had an increase in a contact dependent manner (Figs $1 \mathrm{~A}$ and $\mathrm{B}$ ).

Additionally, after 48 hours of LPS stimulation, the caspase- 3 activity was found to be higher in all culture conditions compared to non-stimulated cultures (data not shown). LPS treatment showed higher levels of caspase- 3 activity on Caco- 2 epithelial and THP-1 monocyte co-culture condition without cell contact compared to the cell-cell contact one (Fig. 2).

\section{Protein expressions of MAPK signalling pathway}

The levels of MAPK intracellular signalling pathway proteins (total or phosphorylated) JNK, Jun, ERK, and p38 were measured by ELISA in Caco- 2 and THP-1 cells co-culture model after 24 hours of LPS treatment (Fig. 3). Overall these proteins slightly increased after 24 hours of LPS stimulation compared to the control groups (data not shown). Regarding to different culture conditions after 24 hours of LPS stimulation, both total p38, phosphorylated p38 and total jun were found to be higher in co-culture model in cell-cell contact manner compared to the cells alone and noncontacted co-culture model, whereas the phosphorylated jun had a trend in the same condition (Figs 3, A-B, E-F).

Protein expressions and related cytokines of $N F-\kappa B$ signalling pathway

After 24 hours of LPS treatment on Caco-2 epithelial and THP-1 monocyte cells co-culture model, total and phosphorylated $\mathrm{NF} \kappa \mathrm{B}$, phosphorylated I $\mathrm{B}$ were measured in the cell lysates, and 24 and 48 hours after incubation with TNF- $\alpha$ and IL-10 were assessed from the cell supernatants by ELISA. Overall, the expressions of them indicated an activation profile after LPS stimulation compared to the non-stimulated culture conditions (data not

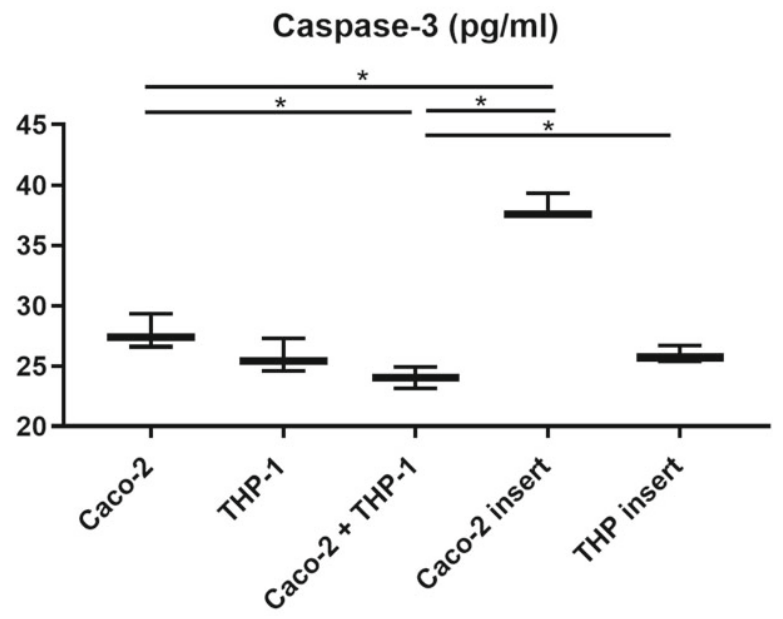

Fig. 2. The effect of LPS stimulation on caspase-3 in Caco-2 epithelia and THP-1 monocyte co-culture model. The horizontal bars indicate the medians of the results within each column. The results of the statistical analysis are indicated as * $p<0.05$.


and phosphorylated $\mathrm{I} \kappa \mathrm{B}$ showed an increase in the co-culture condition with inserts compared to the cell-cell contact conditions, whereas cell-cell contact conditions were also lower than the cells alone in total NFKB (Figs 4A and B). Additionally, after LPS stimulation Caco-2 cells and THP-1 cells co-culture had higher activation of phosphorylated $\mathrm{I} \kappa \mathrm{B}$ than the non-contacted cell culture models (Fig. 4C).

After 48 hours of LPS treatment, secretion of the proinflammatory cytokine TNF- $\alpha$ was slightly increased in Caco- 2 epithelial cells - THP-1 monocyte cells co-culture condition, which was dependent on the cell contact (Fig. 5A). On the contrary, IL-10 secretion did not show prominent features regarding the co-culture conditions (Fig. 5B). 
(A)

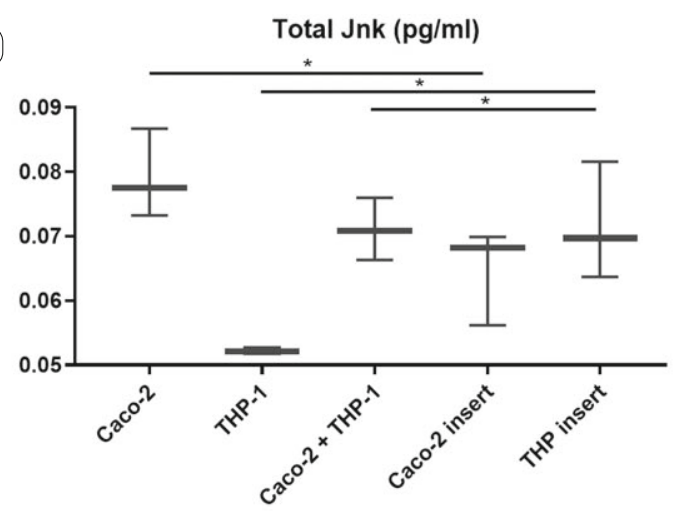

(C)



(E)



(G)



(B)

Phosphorylated Jnk (pg/ml)

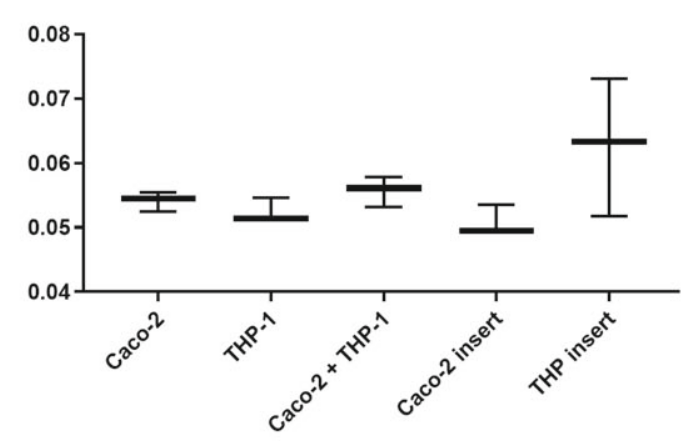

(D)

Phosphorylated Jun (pg/ml)

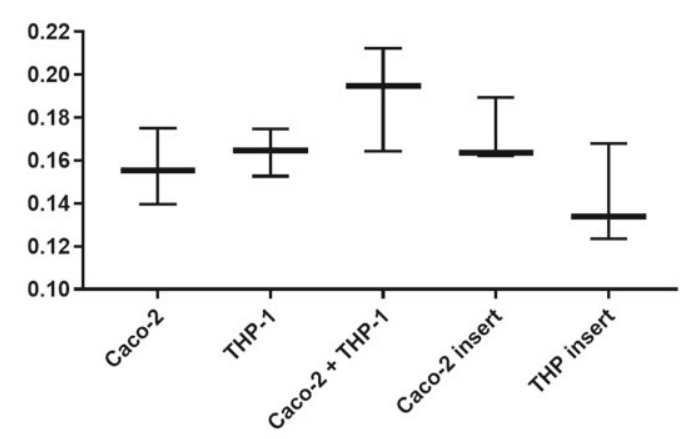

(F)

Phosphorylated erk (pg/ml)

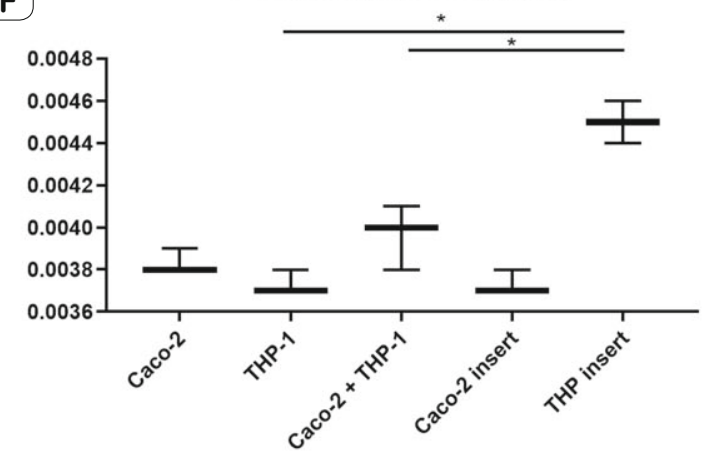

(H)

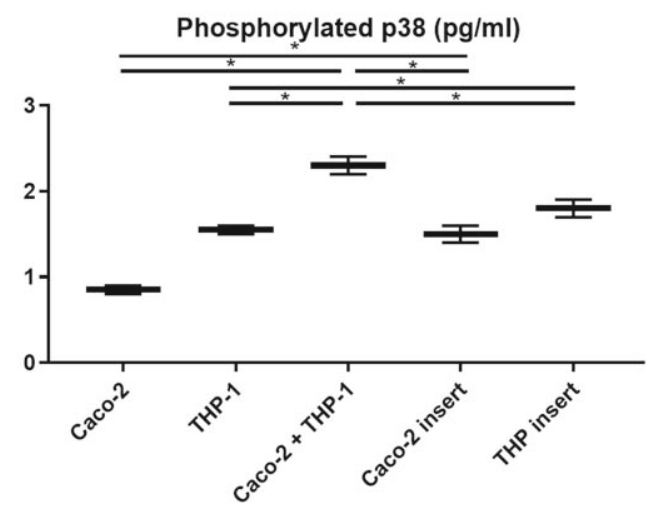

Fig. 3. The effect of LPS treatment on MAPK pathway in Caco-2 and THP-1 cell co-culture model. Activation of (A) total Jnk, (B) phosphorylated Jnk, (C) total Jun, (D) phosphorylated Jun, (E) total erk, (F) phosphorylated erk, (G) total p38 and (H) phosphorylated p38 on different co-culture conditions after 24 hours of LPS stimulation. The horizontal bars indicate the medians of the results within each column. The results of the statistical analysis are indicated as * $p<0.05$. 
A

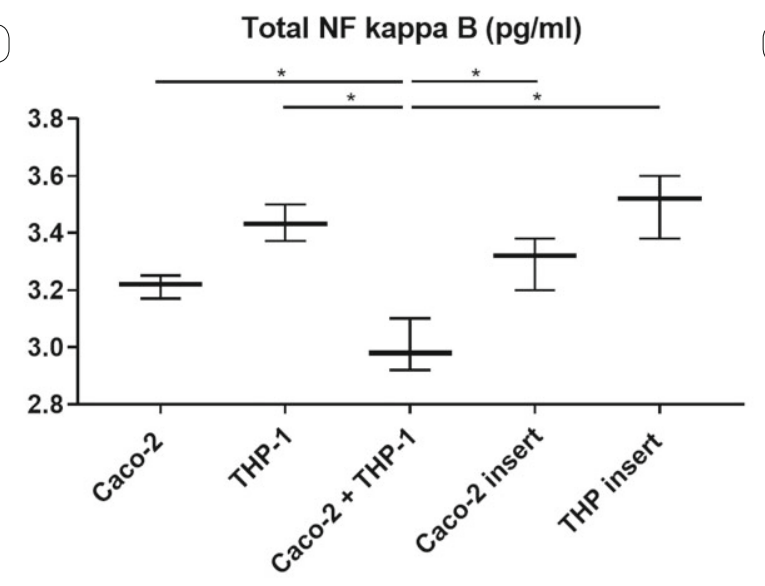

(B)

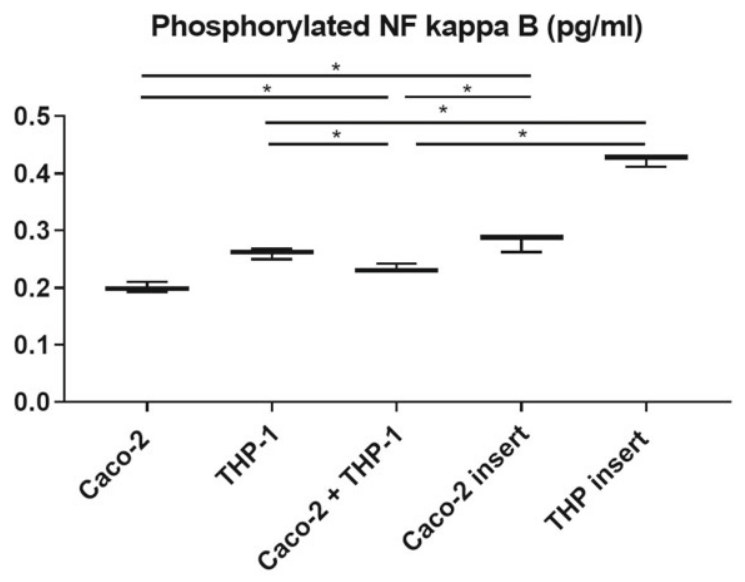

C

Phosphorylated I kappa B (pg/ml)

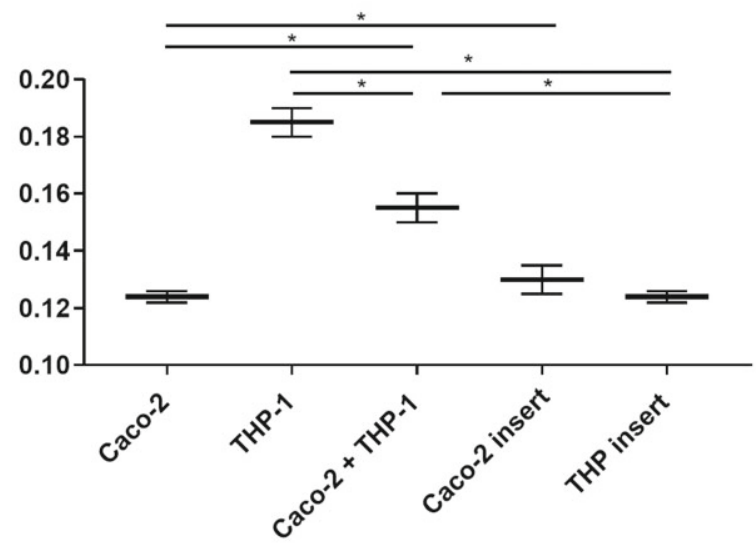

Fig. 4. The effect of LPS treatment on NFאB in Caco-2 epithelia and THP-1 monocyte co-culture model. Activation of (A) total NFкB, (B) phosphorylated NFKB (C) and phosphorylated IKB on different co-culture conditions after 24 hours of LPS stimulation. The horizontal bars indicate the medians of the results within each column. The results of the statistical analysis are indicated as * $p<0.05$.

(A)

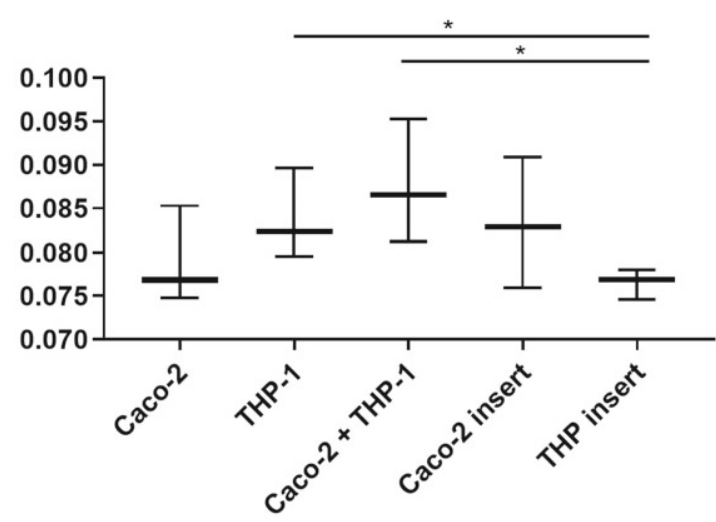

(B)

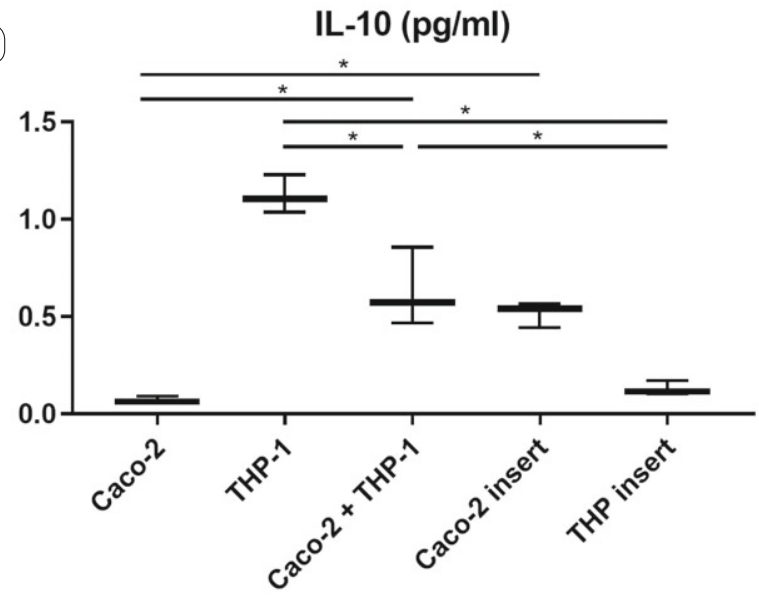

Fig. 5. The effect of LPS treatment on cytokine secretions in Caco-2 epithelia and THP-1 monocyte co-culture model. Activation of (A) TNF- $\alpha$ and (B) IL-10 on different co-culture conditions after 24 hours of LPS stimulation. The horizontal bars indicate the medians of the results within each column. The results of the statistical analysis are indicated as * $p<0.05$. 


\section{Discussion}

The role of the epithelial cells in the regulation of the innate and adaptive immune response on mucosal surfaces has been shown in many studies (20-23). Co-culture models are important for explaining the pathologies especially in the gastrointestinal tract, where the immune cell - epithelial cell interactions are involved $(24,25)$. Interactions between epithelial and immune cells are complex and poorly understood. To better appreciate the signalling pathways at the millieu of epithelial cells and macrophage interaction, with a prior stimulation of LPS, Caco-2 epithelial and THP-1 monocyte-like macrophage cells co-culture model allowed us to assess the cell proliferation, inflammatory responses and apoptosis in different types of culture conditions, which was cells alone, together contact and non-contact dependent manner.

The MAPK pathways were involved in many pathological conditions, including cancer and inflammatory diseases. MAPK transmits external signals into the cells, thus generates the appropriate cellular responses such as: cellular proliferation, differentiation, or cellular survival $(8,26)$. In our study, in terms of cell proliferation and apoptosis, THP-1 monocyte-like cells represented higher levels of JNK, jun and p38 proliferation related protein levels in the presence of Caco-2 epithelia in a contact dependent manner, whereas the filter-separated culture condition led to an increase in phosphorylated p53 suggesting apoptosis in THP-1 cells. Similarly, Caco-2 epithelial cells showed higher levels of jun and p 38 in the presence of the contact with THP-1 monocytes, while the apoptosis related molecule caspase- 3 increased in the non-contact culture condition.

It has been well known that $\mathrm{NF}-\kappa \mathrm{B}$ regulates the proliferation, activation, differentiation and effector function of inflammatory cells and controls also many functions of macrophages and epithelial cells. Thus, it is not suprising that, deregulated NF- $\kappa B$ activation has been shown as a hallmark of chronic inflammatory diseases (11). Additionally, Striz et al. (9) demontrated in respiratory epithelial cell - THP-1 monocytes co-culture that, epithelial cells regulate the expression of multiple genes of NF- $\kappa B$ dependent signaling pathways and lead to the immune response of macrophages. In our study, the changes in NF- $\mathrm{BB}$ signalling pathways and inflammation related cytokine responses of epithelial cells - monocytes co-culture condition revealed an inflammatory situation after LPS stimulation. The contact dependent co-culture condition was characterized with high phosphprylated $\mathrm{NF}-\kappa \mathrm{B}$ and TNF- $\alpha$ levels, whereas low I- $\kappa \mathrm{B}$ and IL-10 levels. In another study investigating the cellular interactions between macrophages and intestinal epithelia, responsible for the regulation of the intestinal epithelial barrier, it has been shown that the expression of COX-2 and NF- $\mathrm{kB}$ p 65 proteins has increased and that this activation has stimulated the secretion of inflammatory cytokines such as IL-1 $\beta$ and TNF- $\alpha$ from LPS stimulated THP-1 cells (27). In many cellular and experimental models, it has been seen that the inflammatory cytokines, such as IL-1, TNF- $\alpha$, IL-6 playing an integral role in the intracellular level of MAPK and NF-kB signalling pathways (28-30).
We conclude that the epithelial cells and monocytes co-culture model regulate the proliferation of cells and the expression of proinflammatory pathways and cytokines, whereas apoptosis and the anti-inflammatory response were delayed. Further studies will contribute to a better understanding of signalling pathways, to elucidate the pathogenesis of many inflammatory diseases progressing with inflammation and the usage of these signalling pathways as pharmaceutical targets for treatment.

\section{References}

1. Strober W. Interactions between epithelial cells and immun cells in the intestine. Ann NY Acad Sci 1998; 1: 37-45.

2. Ganz T. Epithelia: Not just physical barriers. Proc Natl Acad Sci 2002; 99 (6): 3357-3358.

3. Peterson LW, Artis D. Intestinal epithelial cells: regulators of barrier function and immune homeostasis. Nat Rev Immunol 2014; 14: 141-153.

4. Noel G, Baetz NW, Staab JF et al. A primary human macrophage enteroid co-culture model to investigate mucosal gut physiology and hostpathogen interactions. Scientific Reports 2017; 7: 45270.

5. Si-Tahar M, Touqui L, Chignard M. Innate immunity and inflammation-two facets of the same anti-infectious reaction. Clin Exp Immunol 2009; 156(2): 194-198.

6. Mori A, Satsu H, Shimizu M. New model for studying the migration of immune cells into intestinal epithelial cell monolayers. Cytotechnology 2003; 43: 57-64.

7. Farberman MM, Hoffmann JW, Ryerse JS, deMello DE. Diffusible signal to murine alveolar macrophages from lipopolisaccaride and Escherichia coli-stimulated lung type II epithelial cells. Inflamm Res 2004; 53: 475-483.

8. Zhang W, Liu HT. MAPK signal pathways in the regulation of cell proliferation in mammalian cells. Cell Res 2002; 12(1): 9-18.

9. Striz I, Brabcova E, Kolesara L et al. Epithelial cells modulate genes associated with NF kappa B activation in co-cultured human macrophages. Immunobiology 2011; 216 (10): 1110-1116.

10. Ji Q, Wang YL, Xia LM, Yang Y, Wang CS, Mei YQ. High shear stress suppresses proliferation and migration but promotes apoptosis of endothelial cells co-cultured with vascular smooth muscle cells via downregulating MAPK pathway. J Cardiothor Surg 2019; 14: 216.

11. Liu T, Zhang L, Joo D, Sun SC. NF-кB signaling in inflammation. Signal Transduction and Targeted Therapy 2017; 2, e17023. DOI: 10.1038/ sigtrans.2017.23.

12. Pires BRB, Silva RCMC, Ferreira GM, Abdelhay E. NF-kappaB: Two Sides of the Same Coin. Genes 2018; 9 (1): 24.

13. Steinhauer J. Co-culture Activation of MAP Kinase in Drosophila S2 Cells. Methods Mol Biol 2017; 1487: 235-241.

14. Gordon S. Mononuclear phagocytes in immune defence. In: Male D, Brostoff J, Roth DB (Eds). Immunology. Roitt, USA: Mosby Elsevier, 2006; 181-202.

15. Hyun J, Romero L, Riveron R et al. Human intestinal epithelial cells express interleukin-10 through Toll-like receptor 4-mediated epithelialmacrophage crosstalk. J Innate Immun 2015; 7 (1): 87-101.

16. Liu JJ, Davis EM, Wine $\mathbf{E}$ et al. Epithelial cell extrusion leads to breaches in the intestinal epithelium. Inflamm Bowel Dis 2013; 19 (5): 912-921. 
138-144

17. Biriken D, Albayrak N, Yildiz S, Özenci H. Investigation of bactericidal effect and nitric oxide responses of Caco-2 epithelial cells and THP-1 macrophage cells against Streptococcus pyogenes and Escherichia coli. Mikrobiyol Bül 2009; 43 (3): 373-381.

18. Mosman T. Rapid colorimetric assay for cellular growth and survival: Application to proliferation and cytotoxicity assays. J Immunol Methods 1983; 65 (1-2): 55-63.

19. Bijur GN, Jope RS. Proapoptotic stimuli induce nuclear accumulation of glycogen synthase kinase-3 $\beta$. J Biol Chem 2001; 276: 37436-37442.

20. Albayrak N, Biriken D, Özenci H. Investigation of bactericidal effects of human oral and urinary system epithelial cells against different Escherichia coli strains. Mikrobiyol Bül 2005; 39: 161-167.

21. Albayrak N, Biriken D, Özenci H. Investigation of the bactericidal effect of urinary epithelial cells against different doses of Escherichia coli. Mikrobiyol Bül 2006; 40: 195-200.

22. Karahan ZC, Biriken D, Boyvat A, Dolapçi I, Aksu E, Özenci

H. Functionalpolymorphisms and haplotypes of human Beta Defensin 1 (DEFB1) gene in Behcet's disease patients. Clin Exp Rheumatol 2010; 28: $\mathrm{S} 164$

23. Aksu E, Biriken D, Boyvat A, Özenci. Bactericidal effects of uroepithelial cells against Streptococcus pyogenes and Escherichia coli in patients with Behçet's disease and in healty controls. Turkiye Klinikleri J Med Sci 2013; 33 (3): 673-677.
24. Biriken D, Albayrak N, Yildiz S, Özenci H. Investigation of bactericidal effect and nitric oxide responses of Caco-2 epithelial cells and THP-1 macrophage cells against Streptococcus pyogenes and Escherichia coli. Mikrobiyol Bül 2009; 43(3): 373-381.

25. Akarsu G, Biriken D, Karahan ZC. Investigation of Cytokine Responses and Variations in the Expression of Beta-defensin-3 of Caco-2 Human Colon Epidermal Adenocarsinoma and THP-1 Human Leukemia Monocyte Cell Lines in Response to Toxoplasma gondii Under Various Conditions. Mikrobiyol Bül 2017; 51 (2): 156-164.

26. DuShane JK, Mayberry CL, Wilczek MP, Nichols SL, Maginnis MS. JCPyV-Induced MAPK Signaling Activates Transcription Factors during Infection. Int J Mol Sci 2019; 20: 4779.

27. Al-Ghadban S, Kaissi S, Homaidan FR, Naim HY, EL-Sabban ME. Cross-talk between intestinal epithelial cells and immune cells in inflammatory bowel disease. Sci Rep 2016; 6: 297-283.

28. Killeen MJ, Linder M, Pontoniere P, Crea R. NF- $\kappa \beta$ signaling and chronic inflammatory diseases: exploring the potential of natural products to drive new therapeutic opportunities. Drug Discov Today 2014; 19 (4): 373-378.

29. Mantovani A. Molecular pathways linking inflammation and cancer. Curr Mol Med 2010; 10 (4): 369-373.

30. Karin M. Nuclear factor- $\kappa \mathrm{B}$ in cancer development and progression. Nature 2006; 441: 431-436. 